\title{
Discriminating cognitive processes with eye movements in a decision-making driving task.
}

\author{
Sophie Lemonnier \\ Laboratory for road Operations, Perception, Simulators and Simulations, IFSTTAR, France \\ Laboratory of Human and Artificial Cognition, LUTIN, University of Paris VIII, France \\ Roland Brémond \\ Laboratory for road Operations, Perception, \\ Simulators and Simulations, IFSTTAR, France \\ Thierry Baccino \\ Laboratory of Human and Artificial Cognition, \\ LUTIN, University of Paris VIII, France
}

\begin{abstract}
An experiment was conducted in a driving simulator to test how eye-movement patterns evolve over time according to the decision-making processes involved in a driving task. Participants had to drive up to a crossroads and decide to stop or not. The decision-making task was considered as the succession of two phases associated with cognitive processes: Differentiation (leading to a prior decision) and Consolidation (leading to a final decision). Road signs (Stop, Priority and GiveWay) varied across situations, and the stopping behavior (Go and NoGo) was recorded. Saccade amplitudes and fixation durations were analyzed. Specific patterns were found for each condition in accordance with the associated processes: high visual exploration (larger saccade amplitudes and shorter fixation durations) for the Differentiation phase, and lower visual exploration (smaller saccades and longer fixations) for the Consolidation phase. These results support that eye-movements can provide good indices of underlying processes occurring during a decision-making task in an everyday context.
\end{abstract}

Keywords: visual attention, decision making, saccade amplitude, fixation duration, driving

\section{Introduction}

Visual attention is characterized by two types of processing: bottom-up processing is related to the visual characteristics of the scene, and may be modeled by saliency maps (Itti \& Koch, 2001; Koch \& Ullman, 1985; Follet et al., 2010; Bruce \& Tsotsos, 2009); top-down processing depends on prior knowledge, goals and expectancies (Sprague \& Ballard, 2003; Summerfield \& Egner, 2009). Top-down processing is known to have a strong influence on eye movement (Howard et al., 2011; Hayhoe \& Ballard, 2005; Land, 2004; Hayhoe et al., 2003; Henderson \& Hollingworth, 1999; Land et al., 1999; Ballard et al., 1995; Land \& Lee, 1994), which is supported by research on neural mechanisms (Li et al., 2004), while bottom-up processing poorly predicts eye movements in everyday tasks (Rothkopf et al., 2007). Since Yarbus (1967), a number of authors have demonstrated that oculomotor patterns depend on the task (Lipps \& Pelz, 2004; Tatler et al., 2011). Most of these studies have collected eye movements in laboratory conditions, which restrict the generalization of these observations to ecological and complex situations (e.g. driving, playing sports, shopping, serving tea).

Previous studies have considered static tasks, in the sense that task requirement itself does not change dur- ing execution. Consequently, such studies do not reveal possible qualitative changes in gaze patterns over task phases (however, see Ballard et al. (1992, 1995); Mennie et al. (2007), who studied dynamic tasks). It is interesting to test whether it is possible to discriminate different task phases during the course of the task. In addition, in most studies, the decision-making tasks performed by the participants were manipulated by explicit instructions (e.g. lane changing, gap judgment in driving); and these tasks were clearly distinct from one another (e.g. Yarbus' experiment: give the age, remember the clothes or the position of people, estimate material circumstances, etc.). This raises the question whether it is possible to discriminate between the mental states underlying an ongoing task, without changing the stimulus or instruction. Indeed, it would be valuable for everyday eye movement studies.

For these reasons, this paper is focused on the cognitive processes which contribute to the task, and their relationship with eye movements. We have chosen a decision-making task, which consists of two consecutive processes, in a sequential temporal structure. The experimental situation was a crossroads approach in a driving simulator, the decision was to stop or not. Is it possible to discriminate, using oculomotor patterns, the different cognitive processes involved in distinct 
Journal of Eye Movement Research

$7(4): 3,1-14$

task phases, and to investigate whether these patterns change over time?

\section{Decision making and temporal evolution}

In everyday situations, decisions are not always optimal under time pressure. Even if a first decision is made, allowing the appropriate action to start, visual control may be needed in order to check the decision's validity until the action is finished. Experimental evidence of visual control in the course of action has shown for arm and hand movements (e.g. grasping a static target (Goodale, 2011); intercepting a moving target (Brenner \& Smeets, 2011)), as well as driving (Wallis et al., 2007).

In the following, we will consider a decision-making task in a situation with a time course: the decision may occur before action, so that the first decision may be reconsidered until the final decision. This latency introduces a time course of decision-making with two consecutive phases. This type of situation appears in many real-life situations, but is not considered in most decision-making models (general: Kahneman (2002); Eccles \& Wigfield (2002); Gigerenzer \& Gaissmaier (2011); perceptual decision: Geisler (2011); Summerfield et al. (2012); natural tasks: Sprague \& Ballard (2003); under time pressure: Maule et al. (2000); theory of action: Nenkov \& Gollwitzer (2012)). The first decision is labeled "prior decision". The phase before this prior decision correspond to a "Differentiation" process (D). During D, the available information is evaluated and a prior decision is produced as output. The phase after this prior decision correspond to a "Consolidation" process $(\mathrm{C})$. During $\mathrm{C}$, the prior decision is checked, decreasing the uncertainty, and leads to a final decision.

This time course follows the Differentiation and Consolidation (Diff / Con) theory of decision making (Svenson, 1992). The decision-making task is described within this framework as a Differentiation process followed by a Consolidation process; the latter strengthens the prior decision with congruent information (Svenson et al., 2009). This model mainly focuses on social psychology aspects of decision making, and addresses the impact of emotion, stress and attractiveness. Despite difference between the original model of Svenson and its use in a driving context, the vocabulary (D and C) has been preserved.

In the time course of a decision task, the $\mathrm{D}$ process leads to a prior decision. Its aim is to make the best possible decisional choice, considering the current situation. Then, the $C$ process triggers action and validates (or not) this prior decision. Its aim is to check whether the current information is consistent, gradually increasing confidence in the prior decision, until the realization of the action or until confidence level is considered sufficient. In the latter case, if a next decision is needed, a new D process may start (anticipation); otherwise, no
Lemonnier, S., Brémond, R. \& Baccino, T. (2014) Eye movements in a decision-making driving task

decision-making task (and thus, no D or C process) is present any more. In the following, when no decision is required any more, the current activity is described as Baseline (B), in contrast to D and C. D and C have cognitive costs associated with the decision-making task that are added to this baseline, which includes all other processes potentially at work.

In this study, we will specify the $\mathrm{D}$ and $\mathrm{C}$ processes in a specific decision-making task, define their characteristics and those of the associated phases. The challenging issue will then be to discriminate these decision-making processes on the basis of specific eye movement patterns.

\section{The experimental situation: a driver approaching a crossroads}

A number of elements influenced the choice of the experimental situation. Firstly, this study required a delay between prior decision and action (in order to observe C). Moreover, it needed an ecological and complex situation, a central role of perception, a time course, and minimal instructions, the same in all conditions. Indeed, the more complex the situation, the less the gaze is driven by the properties of the visual scene, via bottom-up processing (Foulsham et al., 2011; Land $\&$ Tatler, 2009). For all these reasons, we have chosen a driving situation.

The experimental situation needed also to vary the result of the decision and the time course of the phases (before and after the prior decision). A behavioral observation was also needed in order to record the prior and final decision. The crossroads approach was selected. An important decision has to be made: to stop or not (NoGo vs. Go). If the final decision is to stop, a new decision has to be made: when to restart. The probability of the decision (Go / NoGo) was modulated with the road sign: Stop, Priority and Give Way (see Fig. 1 for an illustration of the sequential temporal structure).

In the Stop sign condition, the prior decision NoGo was imposed by a road sign ${ }^{1}$. The action does not depend on interaction with others vehicles. The probability to have a collision and to change the prior decision is very low. Therefore, confidence in the prior decision is high, and the $C$ process is almost not needed. In this case, the process associated with the decision-making task is expected to be first B, then D in order to anticipate the next decision (when to restart).

In the Priority sign condition, the prior decision Go was also suggested by the road sign. The action implies

\footnotetext{
${ }^{1}$ In the data analysis, we have considered the last $150 \mathrm{~m}$ of the crossroads approach: the road signs were located at this distance, which is consistent with the French regulation. At this point, $150 \mathrm{~m}$ before the crossroads, the prior decision has already been made in the Stop road-sign condition (NoGo) and in the Priority sign condition (Go), but not in the Give Way condition (see Fig. 1).
} 
Journal of Eye Movement Research

$7(4): 3,1-14$

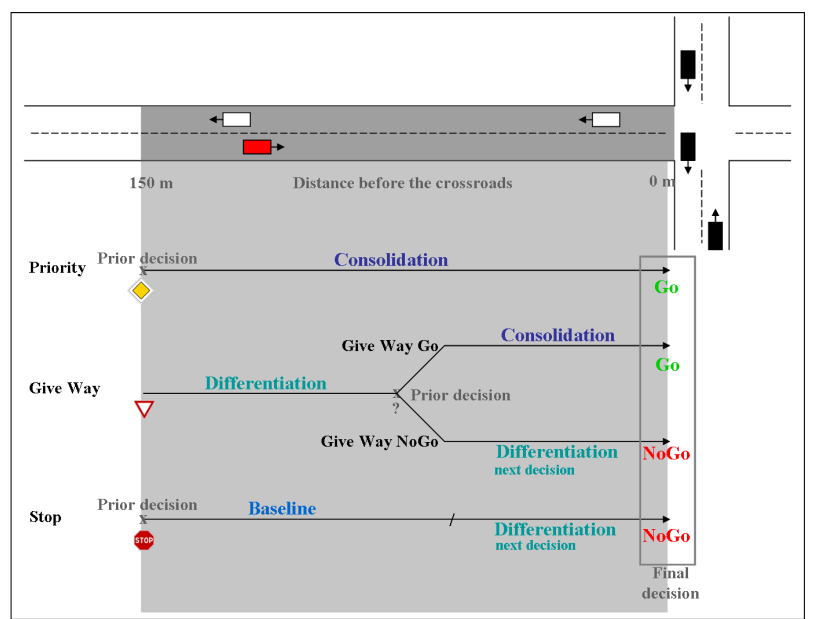

Figure 1. An approach of a crossroads and the prediction of cognitive processes. Top: bird's view of the $150 \mathrm{~m}$ before the crossroads; the participant's car is in red, the traffic cars in black, and control cars in white (to increase immersion feeling). Bottom: predictions about the prior and final decisions, and the associated processes fore the three road sign conditions.

some level of collision risk (if other drivers do not respect the priority). When approaching the crossroads, information about the other driver's behavior increases the level of confidence and consolidates the prior decision. Therefore, the $\mathrm{C}$ process is expected.

In the Give Way sign condition, the prior decision is not obvious, so that the first process is $\mathrm{D}$. Then, if the prior decision is NoGo, the $\mathrm{D}$ process can be replaced by a new $\mathrm{D}$ (anticipation) as in the Stop condition. If the prior decision is Go, the next process is $\mathrm{C}$ as in the Priority condition.

\section{Cognitive processes and eye movements}

Visual search is characterized by scanpaths, resulting in sequences of fixations and saccades (Goldberg \& Kotval, 1999). Chapman \& Underwood (1998) showed that fixation durations decrease and saccade amplitudes increase when visual search increases (see also Rayner (1998); Crundall \& Underwood (2011)).

To decide whether to stop or not, saccade amplitudes and fixation durations (visual exploration ${ }^{2}$ ) are related to estimates of speed, distance and gap size of the oncoming vehicles, based on the information of color, shape, texture and rate of frontal surface expansion (Hancock et al., 1991). This information is supposed to be shared by both the $\mathrm{D}$ and $\mathrm{C}$ processes, the difference between them being the amount of visual exploration.

To use the vocabulary of Sprague \& Ballard (2003), this information is relevant for the decision making task, by reducing uncertainty (e.g. depending on the road sign): "eye movements serve to reduce uncer-
Lemonnier, S., Brémond, R. \& Baccino, T. (2014) Eye movements in a decision-making driving task

tainty about environmental variables that are relevant to behavior" (cited from Sprague \& Ballard (2003), p. 2). Accordingly, the possibility of decreasing the uncertainty is higher during a $\mathrm{D}$ process than during a $\mathrm{C}$ process, thus visual exploration should be higher during a $\mathrm{D}$ process (i.e. larger saccade amplitudes and shorter fixation durations). This is consistent with the driver behavior theory of Näätänen \& Summala (1974): the information obtained during the $\mathrm{D}$ process modifies the expectancy, which will be more specific after the prior decision, during the $\mathrm{C}$ process. This supports a decrease of visual exploration during the $C$ process.

We therefore hypothesized that D, C and B would result in a hierarchy of visual exploration, ranging from more active in D than in C, and in C than in B. According to this hierarchy, the following predictions may be expected: fixation durations should be shorter during $\mathrm{D}$ compared to $\mathrm{C}$ process, and during $\mathrm{C}$ compared to $\mathrm{B}$; and saccade amplitudes should be higher in a D process than in a $C$ process, and in a $C$ process than in a B.
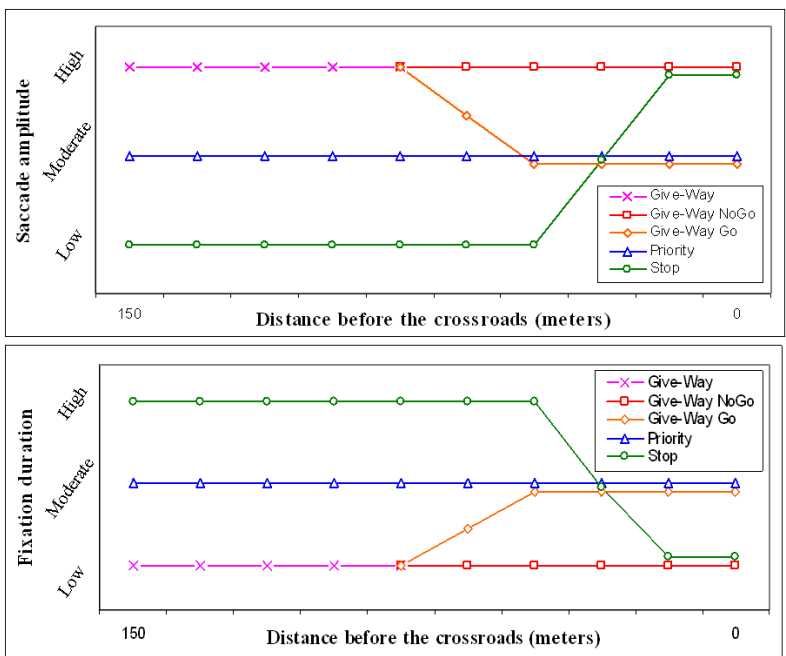

Figure 2. Prediction of oculomotor pattern during the approach of the crossroads, starting at $150 \mathrm{~m}$. Top: Relative predicted value of the saccade amplitudes. Bottom: Relative predicted value of the fixation durations.

To summarize, we link the level of visual exploration with the cognitive processes (see Fig. 2 for an illustration of our oculomotor behavior predictions). The visual exploration is expected to be low in the beginning of the Stop condition (B), and then to increase (D pro-

\footnotetext{
2 "Visual search" is associated in the vision science literature to a detection task. In order to avoid any confusion with the visual search involved in the driving task, which is considered in this study, we use "visual exploration" instead, to denote the combination of fixation durations and saccade amplitudes. Thus, a decrease of fixation durations and an increase of saccade amplitudes will be referred to as an increase of visual exploration.
} 
Journal of Eye Movement Research

$7(4): 3,1-14$

cess). Moderate visual exploration is expected in the beginning of the Priority condition (C process), lowering to a baseline level while evidence is accumulated for the final decision. In the Give Way condition, a high visual exploration is expected (D process) until the prior decision. In the Give Way NoGo condition, this first phase is followed by a new sequence of high visual exploration (D process). In the Give Way Go condition, a moderate visual exploration ( $C$ process) is expected following the prior decision.

\section{Method}

\section{Participants}

Thirty-four participants (29 men and 5 women) with normal or corrected-to-normal vision, took part in the experiment. They all had at least three years of driving experience $(\mathrm{M}=15, \mathrm{SD}=12)$, and their average age was 34 years $(\mathrm{SD}=12)$.

\section{Material}

Virtual environment and scenario. The virtual environment was created with the Realax software. It was displayed at scale 1:1, and computed for the viewpoint of the participant (1.15 $\mathrm{m}$ above the simulated ground). It represented a two-lane highway in a rural environment, with a simplified landscape which helped to reduce bottom-up attention. The road was straight and crossed a new road at right angle every kilometer. The crossroads were announced $150 \mathrm{~m}$ beforehand with a road sign (see Fig. 3). The road markings at the crossroads were consistent with the road signs.

\begin{tabular}{|c|c|c|}
\hline Stop & Give Way & Priority \\
\hline $\begin{array}{c}\text { The driver should } \\
\text { stop and let } \\
\text { traffic pass. }\end{array}$ & $\begin{array}{c}\text { The driver should } \\
\text { let traffic pass, but } \\
\text { it is not required } \\
\text { to stop (yield sign). }\end{array}$ & $\begin{array}{c}\text { The driver has } \\
\text { right of way, he } \\
\text { must not yield } \\
\text { to any vehicle. }\end{array}$ \\
\hline
\end{tabular}

Figure 3. Road signs used in the experiment, with their visual representation and meaning, according to French law.

A driving simulator software architecture (ARCHISIM) was used to introduce traffic in this virtual environment via two computers. In order to improve the sense of immersion, some vehicles were simulated coming along the highway in the opposite direction. At the crossroads, some vehicles were simulated on both ways crossed by the driver (see Fig. 1 , Top). These vehicles followed the priority rules. In order to modulate the stopping decision in the Give Way conditions, three levels of traffic density were
Lemonnier, S., Brémond, R. \& Baccino, T. (2014) Eye movements in a decision-making driving task

implemented: no vehicle, few vehicles (on average spaced $45 \mathrm{~m}$ from each other), or many vehicles (on average spaced $22.5 \mathrm{~m}$ from each other). The three levels of traffic density were also present in the Stop and Priority conditions, to have equivalent visual environments. In case of collision with other vehicles, the participant's car could go through.

Apparatus. The driving simulation experiment (see Fig. 4) was displayed via two computers and five video-projectors, on five screens of $1.85 \mathrm{~m}$ wide and $2.55 \mathrm{~m}$ high (resolution: $1024 \times 1280$ pixels for each video-projector; response time: $60 \mathrm{~Hz}$, consistent with the $60 \mathrm{~Hz}$ simulation update rate). The distance between the participants' heads and the screens (at 90 $)$ was $1.70 \mathrm{~m}$. All five screens formed together an angle of $275^{\circ}$ (see Fig. 4, right). The simulator included a gearbox, a wheel with force feedback, three pedals, a dashboard with speed and engine speed, and a model of a combustion engine.
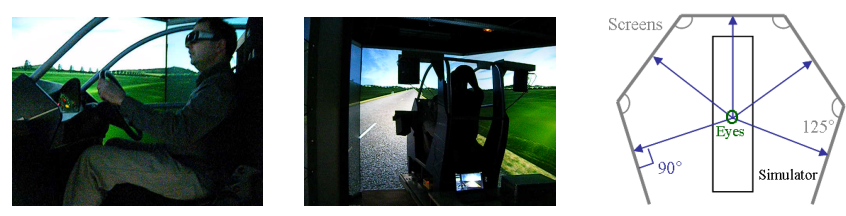

Figure 4. The fixed-based driving simulator. Left: lateral view of the driver equipped with SMI Eye Tracking Glasses; Middle: view from behind; Right: design of the five screens around the driver.

The eye tracking system was SMI (SensoMotoric Instruments) Eye Tracking Glasses (weight: $75 \mathrm{~g}$ ). It included a scene camera (resolution: $1280 \times 960$ pixels, $24 \mathrm{fps}$ ) and two cameras capturing the driver's gaze with a tracking range of $80^{\circ}$ horizontal and $60^{\circ}$ vertical. This system had a gaze position accuracy of $0.5^{\circ}$ (according to the manufacturer), and a sampling rate of $30 \mathrm{~Hz}$. The data was processed using BeGaze (SMI software, version 3.1.77). The fixations were calculated with a dispersion based algorithm, whose parameters were: $80 \mathrm{~ms}$ for minimum fixation duration and 100 pixels $\left(4.69^{\circ}\right)$ for the maximum dispersion. The saccades were then derived from these fixations. This concerns eye movements relative to the head. Head movements were not recorded, which means that gaze shifts relative to the scene may have been much larger in amplitude.

All computers were synchronized with the Absolute Time Server software (two for the environment, two for the simulation and one for the eye tracker).

\section{Procedure}

Once the eye tracker was calibrated (with three points of calibration on anchor locations displayed on the central screen), the participants ran a learning 
Journal of Eye Movement Research

$7(4): 3,1-14$

phase to get accustomed to the driving simulator and to the eye tracking glasses. Then, the main part of the experiment began. Calibration was checked throughout the experiment, and corrected when needed. The participants' task was to drive straight and to cross the crossroads, while taking the road signs into account and avoiding accidents. The instruction was to drive as they would on the same road in reality. The experiment consisted of 45 counter-balanced trials (3 road signs, 3 traffic levels and 5 repetitions), divided up in three blocks (mean duration per block $=17^{\prime} 26^{\prime \prime}, \mathrm{SD}=3^{\prime} 46^{\prime \prime}$ ). After the experiment, the calibration of the eye tracker was checked again. The experiment lasted about one hour.

\section{Analysis}

The hypotheses focused on the crossroads approach. Therefore, the analyses were restricted to the last $150 \mathrm{~m}$ before the crossroads. The oculomotor variables were the fixation durations and the saccade amplitudes. In addition to mean values, our hypotheses were also tested on the slope (mean increase/decrease rate of the saccade amplitudes and fixation durations with respect to the distance to the crossroads). These slopes relate closely to the evolution of the processes over time.

To test our predictions about eye movements with respect to the processes of the decision-making task, preliminary analyses were needed to construct two factors: the Driving Situation and the Position.

The Driving Situation factor describes the various situations the driver could be in during the time course of the crossroads approach (see Fig. 1). It depends on the anticipated stopping behavior ${ }^{3}$ and on the road sign. A NoGo behavior was expected in the Stop condition and a Go behavior in the Priority condition. In the Give Way conditions, the Go and NoGo behaviors (and the associated processes) were both expected. In order to get both decisions, three levels of traffic density were encountered, with "no vehicle" to induce mainly a Go behavior, "many vehicles" to induce mainly a NoGo behavior and "few vehicles" to induce both. The modalities of the Driving Situation factor were Stop with NoGo behavior, Priority with Go behavior, Give Way with Go and with NoGo behavior (GW-Go and GW-NoGo). A descriptive analysis was conducted in order to control that the road sign and traffic density induced the expected stopping behavior, and that the Driving Situation factor makes sense.

The Position factor describes the two phases, Before and After the prior decision in the Give Way condition. It depends on the moment of this prior decision. To identify this moment, the data was cut into 10 road segments depending on the driver's position with respect to the crossroads. Each road segment had a length of $15 \mathrm{~m}$. We postulated that when the driver accelerates significantly in the GW-Go conditions, the prior decision has been made. Thus, it was possible to estimate
Lemonnier, S., Brémond, R. \& Baccino, T. (2014) Eye movements in a decision-making driving task

where the data should be split into two road sections, Before and After this prior decision. Newman-Keuls tests were used to determine significant changes in the gas pedal data comparing the 10 road segments with each other.

Then, the same split was applied to the other conditions, to obtain two data subsets, Before the prior decision with three modalities of the Driving Situation (Give Way, Stop and Priority) and After with four modalities (GW-Go, GW-NoGo, Stop and Priority). For each section, Before and After, two one-factor analyses of variance (ANOVA) were computed on mean values, two more on slopes.

All fixation durations and saccade amplitudes greater than the mean value plus $2 \mathrm{SD}$ were filtered out (respectively above $0.8 \mathrm{~s}$ and above $52.28^{\circ}$ ), which removed $4.07 \%$ of fixation data and $5.26 \%$ of saccade data. The saccade amplitudes were not normally distributed. A logarithmic transform was applied before statistical analysis, leading to a normal distribution. The significance level was set to .05 , but, in order to be consistent with Johnson (2013), p-values are given when $p>.001$.

\section{Results}

\section{Preliminary analyses}

We wanted to link the level of visual exploration with the cognitive processes (D, C and B) associated with a decision-making task (see Fig. 1 and 2). For this, two factors were defined. The first one was the Driving Situation, with Give Way (conditionally to the prior decision: Go or NoGo), Stop and Priority conditions. The second factor was the Position at the crossroads, Before and After the Go/NoGo prior decision in the Give Way condition.

The Driving Situation factor. The expected link was checked between, on the one hand, the Driving Situation factor and on the other hand, the stopping behavior, the road sign and the traffic density (see Tab. 1). As expected, there was no stop behavior in the Priority condition. In some trials, participants did not stop in the Stop condition (13 trials out of 483); these trials were removed from the analysis. In the Give Way condition, there were 216 trials with stop behavior and 274 with no-stop behavior. These values are of the same order of magnitude and are clearly related to the traffic density, showing that modulating the traffic density resulted in two sets of decision, Go and NoGo, with roughly the same size.

The Position factor. Newman-Keuls tests were conducted in order to compare the mean gas pedal acceleration, for all pairs of $15 \mathrm{~m}$ road segments, in the GW-

${ }^{3}$ The stopping behavior was annotated a posteriori from the behavioral data. 
Journal of Eye Movement Research $7(4): 3,1-14$

\begin{tabular}{lccc|ccc}
\hline Traffic & \multicolumn{3}{c|}{ NoGo } & \multicolumn{3}{c}{ Go } \\
\cline { 2 - 7 } density & GW & P & S & GW & P & S \\
\hline No & 7 & 0 & 153 & 155 & 163 & 10 \\
\hline Few & 73 & 0 & 157 & 93 & 162 & 2 \\
\hline Many & 136 & 0 & 160 & 26 & 163 & 1 \\
\hline
\end{tabular}

Table 1

Number of trials with stop at the crossroads (NoGo decision) and without stop (Go decision) with respect to the road sign (Give Way: GW; Priority: P; Stop: S) and traffic density (No, Few or Many vehicles). The total number of trials is 1461.

Go condition (see Appendix A, in italics). No difference appeared until $60 \mathrm{~m}$, where significant differences appeared when comparing the first $(p=0.025)$, second $(p=0.025)$, third $(p=0.013)$ and fourth $(p=0.019)$ road segments to the seventh $(60-45 \mathrm{~m})$. This marker is consistent with the gas pedal curve: the push on the pedal increases sharply $60 \mathrm{~m}$ before the crossroads (see Fig. $5)^{4}$.

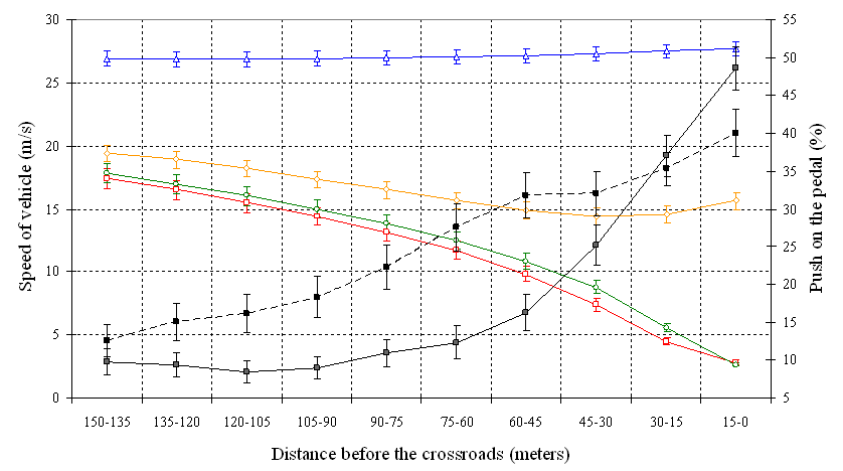

Figure 5. Mean speed for each of four conditions (left axis) and pressing (right axis) on the gas pedal (Give-Way Go condition) and on the brake pedal (Give-Way NoGo condition), as function of the distance to the crossroads. The whiskers represent standards errors. For the vehicle speed, red: GWNoGo, orange: GW-Go, blue: Priority, green: Stop; for the push on the pedal, solid line in black: gas pedal, dash line in black: brake pedal.

The data was thus split into two road sections, at 60 $\mathrm{m}$ from the crossroads. In the following, the first six road segments ( 150 to $60 \mathrm{~m}$ before the crossroads) are referred to as the Before section, and the last four road segments $(60$ to $0 \mathrm{~m})$ are referred to as the After section, according to before and after the prior decision in the Give Way condition.

A systematic interaction was found between the Position and the Driving Situation (see Appendix B), which shows a consistency between the behavioral and oculomotor data (see Fig. 6 for the saccade amplitudes and Fig. 7 for the fixation durations).
Lemonnier, S., Brémond, R. \& Baccino, T. (2014) Eye movements in a decision-making driving task

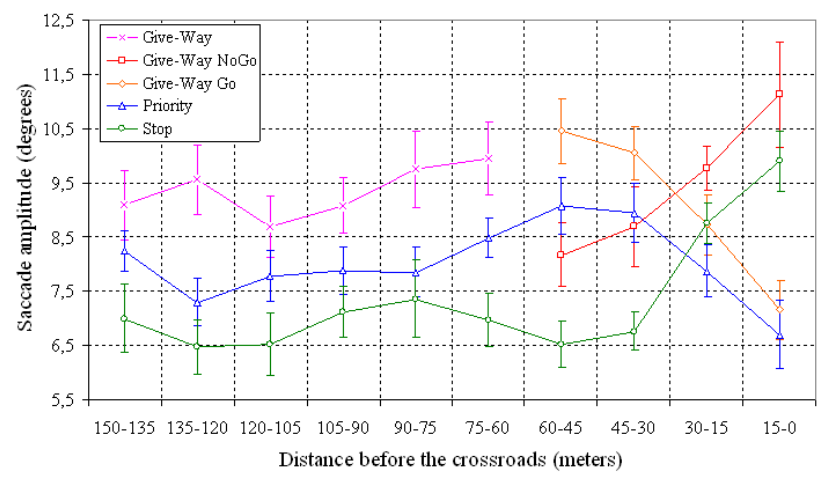

Figure 6. Mean values of the saccade amplitudes as a function of the Position and Driving Situation. The whiskers represent standards errors.

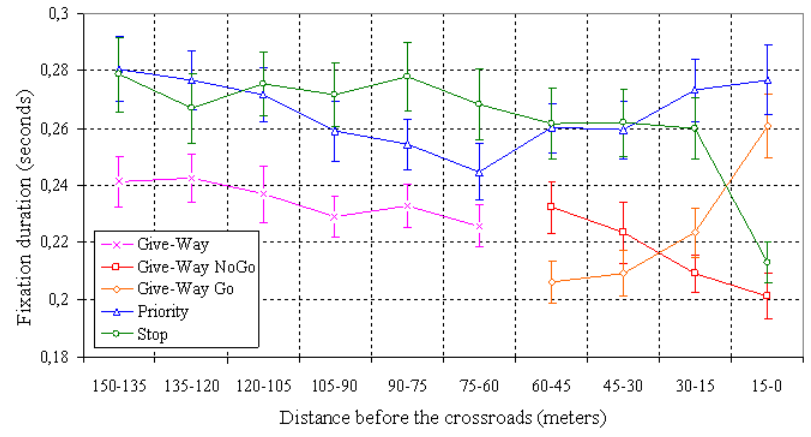

Figure 7. Mean values of the fixation durations as a function of the Position and Driving Situation. The whiskers represent standards errors.

\section{Main results}

On each section, four ANOVA have been conducted for saccade amplitudes and fixation durations, on the mean values (F1) and the mean slopes (F2). In the Before section, three modalities were considered for the Driving Situation: Give Way, Stop and Priority. In the After section, four modalities were considered: GW-NoGo, GW-Go, Stop and Priority. The results of planned contrasts are presented in Appendix C.

The "Before" road section. In the analysis of saccade amplitudes, a significant effect was found on the mean values, $F 1(2,66)=27.365, M S E=0.006, p<.001, \eta^{2}=$ 0.453 , with amplitudes higher in the Give Way condi-

\footnotetext{
${ }^{4}$ Newman-Keuls tests were also conducted on the brake pedal data in the GW-NoGo condition (see Appendix A, in non-italics). Significant changes appear $90 \mathrm{~m}$ before the crossroads, when comparing the first $(p<.001)$, second $(p=0.013)$ and third ( $p=0.045$ ) road segments to the fifth (90-75 meters). The change is less sharp however (see Fig. 5), so that we decided to split the data according to the observed change in the gas pedal behavior.
} 
Journal of Eye Movement Research

$7(4): 3,1-14$

\begin{tabular}{cccrr}
\multicolumn{5}{c}{ Before road section } \\
\hline & Situation & Stop & Priority & GW \\
\hline SA & M & 7.014 & 8.031 & 9.535 \\
\cline { 2 - 5 } & SD & 2.280 & 1.601 & 2.811 \\
\cline { 2 - 5 } & M-slope & 1.087 & 0.974 & 1.976 \\
\cline { 2 - 5 } & SD-slope & 6.254 & 5.037 & 7.121 \\
\hline FD & M & 0.279 & 0.271 & 0.239 \\
\cline { 2 - 5 } & SD & 0.057 & 0.049 & 0.044 \\
\cline { 2 - 5 } & M-slope & 0.007 & -0.076 & -0.042 \\
\cline { 2 - 5 } & SD-slope & 0.094 & 0.082 & 0.089 \\
\hline
\end{tabular}

After road section

\begin{tabular}{|c|c|c|c|c|c|}
\hline \multicolumn{2}{|c|}{ Situation } & Stop & Priority & GWGo & GWNoGo \\
\hline \multirow[t]{4}{*}{$\overline{\mathrm{SA}}$} & $\mathrm{M}$ & 8.222 & 8.412 & 9.146 & 9.592 \\
\hline & SD & 1.881 & 2.035 & 2.423 & 1.569 \\
\hline & M-slope & 12.268 & -7.759 & -10.398 & 11.780 \\
\hline & SD-slope & 12.452 & 15.615 & 10.000 & 20.518 \\
\hline \multirow[t]{4}{*}{ FD } & $\mathrm{M}$ & 0.254 & 0.277 & 0.226 & 0.222 \\
\hline & SD & 0.050 & 0.049 & 0.040 & 0.043 \\
\hline & M-slope & -0.173 & 0.042 & 0.181 & -0.108 \\
\hline & SD-slope & 0.181 & 0.177 & 0.208 & 0.165 \\
\hline
\end{tabular}

Table 2

Means of saccade amplitudes (SA) and fixation durations (FD) according to the Position and Situation experimental factors, in terms of mean values ( $M$; degree for $S A$ and second for FD) and mean slopes (M-slope; degree/150m for $S A$ and second/150m for FD), with the associated standard deviation (SD and SD-slope). The Table on Top refers to the Before position, the bottom one to the After position.

tion than in the Priority condition, and higher in the Priority condition than in the Stop condition (see Tab. 2 for the means and standard deviations, and Fig. 8 for an illustration of contrasts). No significant effect was found on the mean slopes, $F 2(2,66)=0.955, M S E$ $=0.043$.

The analysis of fixation durations showed a significant effect for the mean values, $F 1(2,66)=21.765, M S E$ $=0.001, p<.001, \eta^{2}=0.397$, with durations shorter in the Give Way condition than in the Stop and Priority conditions (no difference was found between these two conditions). A significant effect was also found for the mean slopes, $F 2(2,66)=7.524, M S E=0.008, p=0.001, \eta^{2}$ $=0.186$. The slopes were negatives in the Give Way and Priority conditions and close to zero in the Stop condition (see Tab. 2 for the means and standard deviations, and Fig. 8).

The "After" road section. From the analysis of the saccade amplitudes, a significant effect was found on mean values, $F 1(3,99)=11.552, M S E=0.006, p<.001$, $\eta^{2}=0.259$, with smaller amplitudes in the Priority and Stop conditions compared to the GW-Go and GWNoGo conditions. A significant effect was also found on mean slopes, F2(3,99) $=110.089, M S E=0.206, p<$
Lemonnier, S., Brémond, R. \& Baccino, T. (2014) Eye movements in a decision-making driving task

.001, $\eta^{2}=0.769$. The slopes were positive in the Stop and GW-NoGo conditions, and negative in the Priority and GW-Go conditions (see Tab. 2 for the means and SD, and Fig. 8).

From the analysis of fixation durations, a significant effect was found on mean values, $F 1(3,99)=26.044$, $M S E=0.001, p<.001, \eta^{2}=0.441$, with shorter fixations in the GW-Go and GW-NoGo conditions than in the Stop condition, and shorter in the Stop condition than in the Priority condition. A significant effect was also found on the slopes, $F 2(3,99)=26.344, M S E=0.032, p$ $<.001, \eta^{2}=0.444$. The slopes were positive in the GWGo and Priority conditions, higher in the GW-Go condition, and negative in the GW-NoGo and Stop conditions (see Tab. 2 for the means and SD, and Fig. 8).

The slopes were steeper in the After section (for the saccade amplitudes: $\mathrm{M}=0.0928^{\circ} . \mathrm{m}^{-1}$, SD $=0.0791^{\circ} \cdot \mathrm{m}^{-1}$; for the fixation durations: $\mathrm{M}=$ $\left.0.0027 \mathrm{~s} . \mathrm{m}^{-1}, \mathrm{SD}=0.0024 \mathrm{~s} . \mathrm{m}^{-1}\right)$, compared to the Before section (for the saccade amplitudes: $\mathrm{M}=$ $0.0392^{\circ} \cdot \mathrm{m}^{-1}, \mathrm{SD}=0.0343^{\circ} \cdot \mathrm{m}^{-1}$; for the fixation durations: $\left.\mathrm{M}=0.0017 \mathrm{~s} . \mathrm{m}^{-1}, \mathrm{SD}=0.0014 \mathrm{~s} . \mathrm{m}^{-1}\right)$. This difference suggests a relative stability of the processes during the Before section, while steep slopes indicate process change during the After section.

Summary of the results. In the "Before" road section, Table 2 shows that the highest saccade amplitudes and the shortest fixation durations are found in the Give Way condition (corresponding to the D process), which is congruent with the hypothesis of a higher visual exploration during this process. The difference between the Priority condition ( $C$ process) and the Stop condition (B), with higher amplitudes in C, may be due to a higher level of subjective risk in the Priority condition, related to the absence of interaction associated with the NoGo decision in the Stop condition: with a lower risk level, the process is closer to the Baseline.

In the "After" road section, shorter fixation durations were found in the Stop condition (compared to the Priority condition) which is congruent with the anticipation of the next decision in the Stop condition (new D process). For the saccade amplitudes and fixation durations, no difference was found on the mean values between the GW-Go and GW-NoGo conditions. Figure 6 shows that the curves intersect, suggesting that the slope is a better description of the oculomotor behavior in this case. Indeed, the slopes of saccade amplitudes and fixation durations evolved in the same way in the Stop and GW-NoGo conditions on the one hand, and in the Priority and GW-Go on the other hand. This can be seen as an increase in visual exploration (intensification of $\mathrm{D}$ processes) in the first two (Stop and GW-NoGo), and a decrease of visual exploration in the other two (Priority and GW-Go).

Direct Prediction. Given that our main hypotheses seem to be validated, it was interesting to test whether 

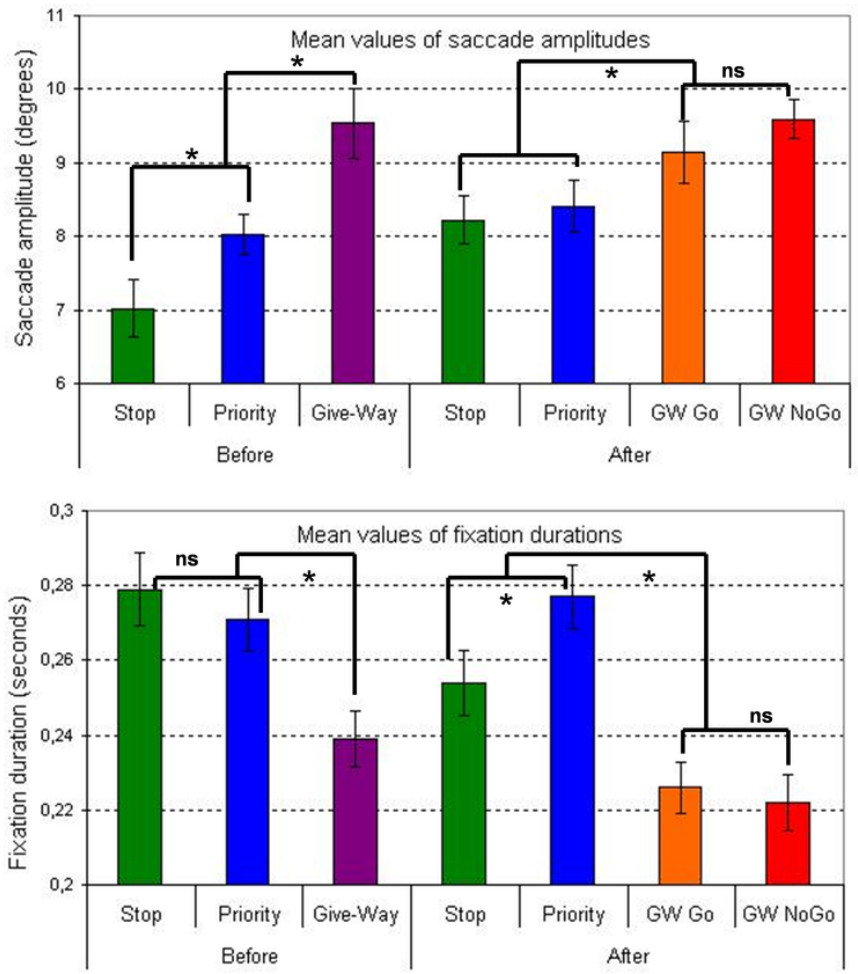

Lemonnier, S., Brémond, R. \& Baccino, T. (2014) Eye movements in a decision-making driving task
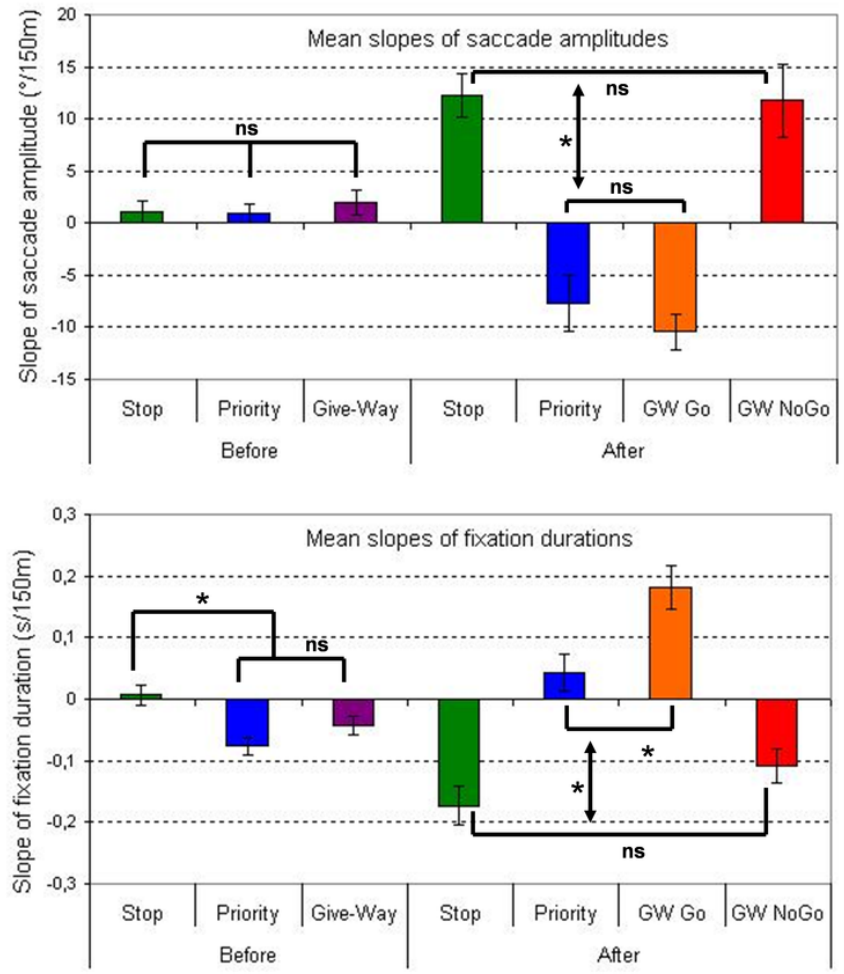

Figure 8. Mean values and slopes of saccade amplitudes and fixation durations according to the experimental factors, Situation and Position. "**": $p$-value of contrast <.05. The whiskers represent standards errors.

it was possible to predict the processes of the decisionmaking task from the eye movement data. Using the same methodology as Greene et al. (2012), we have tested whether a Support Vector Machine (SVM, see Vapnik (1998)) could learn and then estimate, above chance, the subject, the Driving Situation (Stop, Priority, GW-NoGo and GW-Go conditions) or the Position (Before and After sections).

We used the libSVM library, developed by Chang \& Lin (2011), with a Gaussian kernel with $\gamma=2$. Each vector contains four components: saccade amplitude, slope of saccade amplitude, fixation duration and slope of fixation duration. We had two vectors for each participant's trial: one in the "before" and one in the "after" road sections. The data was normalized before the learning phase. The cross-validation used 100 components. The mean performance over these 100 computations was $7.57 \%$ for guessing the subject (chance was $2.94 \%$ ) showing idiosyncratic oculomotor patterns, $46.30 \%$ for the Driving Situation (chance was 25\%) and $71.25 \%$ for the Position (chance was 50\%).

Given that the condition is not equivalent to a cognitive process, we have also tested a simplified association between Position, Driving Situation and process (see Tab. 3), which is derived from the expected dominant process according to the hypotheses under study.

From a 100-components cross-validation, we have

\begin{tabular}{lllll}
\hline & Stop & Priority & GW-Go & GW-NoGo \\
\hline Before & B & C & D & D \\
\hline After & D & C & C & D \\
\hline
\end{tabular}

Table 3

In order to test the SVM, the three cognitive processes are associated to Situation and Position experimental factors: Baseline (B), Consolidation (C) and Differentiation (D).

found a prediction rate of $58.81 \%$ when guessing the cognitive process, which is clearly above chance (33\%). Furthermore, we have also found that using the trends (slopes) in addition to the mean values improves the predictive power of the model: if one discards the slopes, the prediction rate falls to $48.37 \%$.

\section{Discussion}

The current study aimed to establish a link between eye movements and decision-making situations unfolding in time, associated with Differentiation (D) and Consolidation (C). An experimental driving situation was designed, where eye movements were described with saccade amplitudes and fixation durations. An increase of saccade amplitudes and a decrease of fix- 
Journal of Eye Movement Research

$7(4): 3,1-14$

ation durations are reported as an increase of visual exploration. In order to analyze the data, a theoretical framework was introduced for the decision-making task, consistent with the D / C theory (Svenson et al., 2009).

In order to investigate the different processes of the decision-making task (D, C, Baseline - B), the moment and the value of the decision (Go/NoGo) have been identified. Two factors were used in the analyses: the Driving Situation (Stop, Priority, Give-Way Go and NoGo) and the Position of the prior decision in the Give Way condition, with respect to the crossroads.

In the Priority road sign condition, a $C$ process was expected. In the Stop condition, the process was expected to be closer to B. Indeed, the NoGo decision (Stop road sign) does not lead to interactions with others vehicles, unlike the Go decision (Priority road sign). Then, when approaching the crossroads, the driver anticipates the next decision (when to restart) with a new $\mathrm{D}$ process. In the Give Way condition, the D process was first needed, in order to make the prior decision (Go or NoGo). Then, with a NoGo decision, a new D process was expected, as in the Stop condition; with a Go decision, a C process was expected as in the Priority condition.

In the Before section, mean values of saccade amplitudes and fixation durations showed that the highest visual exploration was in the Give Way condition, consistent with a D process. In contrast, visual exploration was the smallest in the Stop condition, confirming a process close to $B$. The mean slopes were quite flat in this section, which means that in each condition, the process was stationary.

In the After section, the visual exploration was found to be more intensive in the Stop condition than in the Priority condition. This switch of patterns compared to the Before section is congruent with the process change in the Stop condition from B to D. Meanwhile, the process seems to be closer to B than to $C$ in the Priority condition. In the GW-NoGo condition, the oculomotor patterns show a D process as before the prior decision. We understand this observation as indicating a new D process, associated with the anticipation of the next decision, as in the Stop condition. In contrast, the visual exploration dramatically lowers in the GW-Go condition, with a profile similar to the Priority condition.

More generally, from the videos, we could see that the gaze was mainly close to the vanishing point of the roadway ("far point" for straight road), in agreement with the literature (Donges, 1978; Land \& Horwood, 1995; Salvucci \& Gray, 2004). The drivers scanned the other cars far away on the left and right when approaching the crossroads in the Give Way and Stop conditions, and looked at the cars waiting for them to pass (at a shorter range) in the Priority condition.

Returning to our main hypothesis, we have found it possible to discriminate visual attention processes at-
Lemonnier, S., Brémond, R. \& Baccino, T. (2014) Eye movements in a decision-making driving task

tached to a decision-making task, with eye movements. Only minimal instructions (the same in all conditions) were needed with respect to the decision-making task. Additional cognitive processes occur in driving, and there are inter-individual differences as well. The validation of our main hypothesis despite this variability suggests that our results are robust. Given that driving is an everyday life activity, we hope that it will be possible to discriminate cognitive processes from oculomotor patterns in other decision-making tasks (Kingstone et al., 2008).

Finally, analyses were conducted in order to predict these cognitive processes from eye movements. Greene et al. (2012) have recently discussed Yarbus' insight that oculomotor patterns may allow the nature of the ongoing task to be predicted, at least better than chance, and surprisingly enough, they found no significant difference when comparing saccade amplitudes and fixation durations in several visual tasks. Conversely, for a different set of tasks, Henderson et al. (2013) give evidence that oculomotor data may be discriminant as far as the visual task is concerned. Our data adds some input to this discussion by predicting the process associated with the decision-making task ( $58.81 \%$, chance is $33 \%$ ). This encouraging result confirms that it is possible in some conditions to evaluate above chance the cognitive process from the current oculomotor patterns, even in a complex situation, such as driving, where cognitive processes cannot be imposed by explicit instructions.

\section{Priority and Stop conditions}

Based on the acceleration profiles in the GW-Go condition, our data was cut into two sections, Before and After the prior decision. An effect of these sections was found in the Priority and Stop conditions, even if we had no prior hypothesis that the associated processes would change $60 \mathrm{~m}$ before the crossroads. In the Priority condition, the process changed from $C$ to $B$, indicating that the decision-making task is over before passing the crossroads, as in the GW-Go condition. In the Stop condition, a process change from B to D was expected (and found), due to the anticipation of the next decision, when to restart. The fast changes of oculomotor patterns in the After section may be due to the fact that when approaching the crossroads, the driver's gaze needs to select information from left and right, at increasingly wider angles, leading to a higher visual exploration. This increase of visual exploration during the time course of the task also occurs in the GW-NoGo condition.

Could we have anticipated that the process changes in the Priority and Stop conditions would occur $60 \mathrm{~m}$ before the crossroads, as in the Give Way conditions? It seems that at this point, the visual information is sufficient to make the prior decision (stopping behavior) or to focus on the next decision (restart behavior). We may hypothesize that the driver, based on prior knowl- 
edge, estimates the best position to make the crossing prior decision which is needed to start action. Indeed, before this position, the visual information may not be sufficient to anticipate correctly the future state of the crossroads; after this position, a stop decision would lead to an uncomfortable brake.

In order to examine this last idea, we considered a scenario where a driver runs at $90 \mathrm{~km} / \mathrm{h}$ (close to the mean speed in our experiment), then brakes at $5 \mathrm{~m} . \mathrm{s}^{-2}$, which is near the limit of comfort. We found a stopping distance of $62.50 \mathrm{~m}$, consistent with this hypothesis. Further experimentation, comparing novice and experienced drivers in the same situation, may help to validate this learning hypothesis. It would also contribute to a better understanding of the link between everyday learning and associated behaviors (eye movements).

\section{Mean slopes of eye-movement data as dependent variables}

We have proposed to use the mean slope of eyemovement data (both saccade amplitude and fixation duration) as dependent variables, and the statistical analysis confirmed the relevance of these variables in our experiment (see also Fig. 6 and Fig. 7). These slopes describe how raw data evolve when approaching the crossroads, and thus make it possible to discriminate between stationary processes (where the raw data remains roughly constant over time) and changing processes (where a global trend can be found in the temporal evolution).

For instance, in the Diff/Con theory of decision making, one can hypothesize that the $C$ process decreases over time, while accumulating evidence for the final decision (Svenson, 1992). This would be consistent with the decrease of visual exploration we have found in the After section, in the Priority and GW-Go conditions. The idea that visual exploration may increase in the $\mathrm{D}$ process when approaching the crossroads (in relation to the next decision) also suggests an evolution of the oculomotor patterns in the time course of the decision-making task.

\section{Conclusion}

The main contribution of this study is that a better understanding of cognitive processes associated to a decision-making task can be extracted from eyemovement patterns in a real-life situation. Trying to determine the cognitive ongoing process from oculomotor patterns in everyday tasks is still in its infancy. Further studies are needed in order to develop a full understanding of the time course of decision making in a greater variety of tasks and scenarios (e.g. in driving situations: overtaking, traffic lights, entering of motorway). Yet, the ability to anticipate human decisions would be highly advantageous in many domains, and enable the development of new aids and systems.

\section{Acknowledgments}

We wish to thank Fabrice Vienne, Jacky Robouant, Stéphane Caro and Isabelle Aillerie for their technical support in the driving simulation experiment; JeanPhilippe Tarel for sharing with us his expertise in machine learning (SVM); Dana Cohen for helping us to improve the manuscript; Régis Lobjois, Otto Lappi and the anonymous reviewer for their valuables suggestions. 
Journal of Eye Movement Research

$7(4): 3,1-14$

\section{Appendix A}

Newman-Keuls test for the

location of the prior decision in the Give Way condition.

\begin{tabular}{ccccccccccc}
\hline Position & 150 & 135 & 120 & 105 & 90 & 75 & 60 & 45 & 30 & 15 \\
classes & -135 & -120 & -105 & -90 & -75 & -60 & -45 & -30 & -15 & -0 \\
\hline $150-135$ & & .474 & .420 & .141 & $\star$ & $\star$ & $\star$ & $\star$ & $\star$ & $\star$ \\
\hline $135-120$ & .874 & & .843 & .711 & .013 & $\star$ & $\star$ & $\star$ & $\star$ & $\star$ \\
\hline $120-105$ & .940 & .909 & & .856 & .045 & $\star$ & $\star$ & $\star$ & $\star$ & $\star$ \\
\hline $105-90$ & .932 & .843 & .828 & & .059 & $\star$ & $\star$ & $\star$ & $\star$ & $\star$ \\
\hline $90-75$ & .610 & .782 & .815 & .822 & & .012 & $\star$ & $\star$ & $\star$ & $\star$ \\
\hline $75-60$ & .498 & .572 & .531 & .574 & .538 & & .041 & .080 & .001 & $\star$ \\
\hline $60-45$ & .025 & .025 & .013 & .019 & .055 & .092 & & .919 & .216 & .001 \\
\hline $45-30$ & $\star$ & $\star$ & $\star$ & $\star$ & $\star$ & $\star$ & $\star$ & & .116 & $\star$ \\
\hline $30-15$ & $\star$ & $\star$ & $\star$ & $\star$ & $\star$ & $\star$ & $\star$ & $\star$ & & .024 \\
\hline $15-0$ & $\star$ & $\star$ & $\star$ & $\star$ & $\star$ & $\star$ & $\star$ & $\star$ & $\star$ & \\
\hline
\end{tabular}

Table A1

Newman-Keuls test ( $p$ values) comparing all pairs of position classes. In italics (bottom left half of the Table): gas pedal data, for the GW-Go condition; in regular (top right half of the Table): braking pedal data, for the GW-NoGo condition. The stars $(\star)$ stand for $p<.001$.

\section{Appendix B \\ Global analysis}

Four $4 \times 2$ ANOVA were conducted on the mean values (F1) and on the mean slopes (F2) of the saccade amplitudes' logarithms and of the fixation durations. These ANOVA tested the effect of the Driving Situation (GW-NoGo, GW-Go, Stop and Priority) and of the Position (Before and After sections).

Significant effects of the Driving Situation were found on the saccade amplitudes, F1(3,99) $=21.38$, MSE $=0.008, p<.001, \eta^{2}=0.393 ; F 2(3,99)=77.33, M S E=$ $0.155, p<.001, \eta^{2}=0.701$. For the Position, a significant effect was found on the mean values, and a marginally non-significant effect on the mean slopes, $F 1(1,33)=$ 6.95, MSE $=0.009, p=0.013, \eta^{2}=0.174 ; F 2(1,33)=4.05$, $M S E=0.205, p=0.052$. Significant interaction effects were found, $F 1(3,99)=16.61, M S E=0.005, p<.001, \eta^{2}$ $=0.335 ; F 2(3,99)=83.85, M S E=0.129, p<.001, \eta^{2}=$ 0.718 (see Fig. 6).

Significant effects of the Driving Situation were also found on the fixation durations, $F 1(3,99)=32.42$, MSE $=0.001, p<.001, \eta^{2}=0.496 ; F 2(3,99)=15.04, M S E=$ $0.019, p<.001, \eta^{2}=0.313$. For the Position, a significant effect was found on the mean values, but not on the mean slopes, $F 1(1,33)=7.54, M S E=0.001, p=0.010$, $\eta^{2}=0.186 ; F 2(1,33)=1.19, M S E=0.028$. Significant interaction effects were found, $F 1(3,99)=12.47, M S E=$
Lemonnier, S., Brémond, R. \& Baccino, T. (2014) Eye movements in a decision-making driving task

$0.001, p<.001, \eta^{2}=0.274 ; F 2(3,99)=24.49, M S E=0.025$, $p<.001, \eta^{2}=0.426$ (see Fig. 7).

A significant difference was found between the two Give Way conditions (Go and NoGo) for the mean values (not for slopes) in the Before section (both for saccade amplitudes and fixation durations). This effect was not expected and more investigations were needed. We found that the effect was due to the "few vehicles" condition. Without these trials, Student's ttest comparing GW-NoGo and GW-Go conditions in the Before section was not significant: $T(66)=-1.754, p$ $=0.436$, for the saccade amplitudes; and $T(66)=-1.754$, $p=0.909$, for the fixation durations.

Trials with "few vehicles" were those where the decision task is more complex. In contrast, in the "many vehicles" condition, the final decision is often to stop (see Tab. 1), while participants almost always passed in the "no vehicle" condition. One explanation could be that people become quickly aware of the higher complexity of the "few vehicles" condition, and depending on their skills, some drivers may have chosen to defer their prior decision. In this case, their visual exploration would have been reduced, as has been observed for both saccade amplitudes and fixation durations. Nevertheless, it is interesting to note that despite of the increased complexity of this task, the results are consistent with our main hypotheses, thus emphasizing their robustness. 
Journal of Eye Movement Research $7(4): 3,1-14$

\section{Appendix C Planned contrasts}

Results of planned contrasts on the ANOVA presented in the Main Results section, which compared the Driving Situations Give Way, Stop and Priority in the Before road section, and the Driving Situations GW-Go, GW-NoGo, Stop and Priority in the After road section.

\begin{tabular}{llrrrr} 
& & Deg & Avg & $F$ & $p$ \\
\cline { 2 - 6 } Before & S vs. P & 1 & 0.13937 & 27.04751 & $<.001$ \\
& Error & 33 & 0.00515 & & \\
\cline { 2 - 6 } & P vs. GW & 1 & 0.02884 & 4.67711 & 0.03791 \\
& Error & 33 & 0.00617 & & \\
\hline After & S vs. P & 1 & 0.02526 & 5.41869 & 0.02620 \\
& Error & 33 & 0.00466 & & \\
\cline { 2 - 6 } & GWGo vs. GWNoGo & 1 & 0.01133 & 1.10373 & 0.30108 \\
& Error & 33 & 0.01027 & & \\
\cline { 2 - 6 } & GW vs. P & 1 & 0.19709 & 62.85244 & $<.001$ \\
& Error & 33 & 0.00314 & & \\
\hline
\end{tabular}

Table C1

Planned contrasts on mean values of saccade amplitudes. Road signs are Stop (S), Priority (P) and Give Way (GW).

\begin{tabular}{llrrrr} 
& & Deg & Avg & $F$ & $p$ \\
\hline \multirow{2}{*}{ After } & S vs. GW NoGo & 1 & 0.04905 & 0.20233 & 0.65579 \\
& Error & 33 & 0.24244 & & \\
\cline { 2 - 5 } & P vs. GW Go & 1 & 0.16382 & 1.046346 & 0.31379 \\
Error & 33 & 0.15656 & & \\
\cline { 2 - 5 } & S/GWNoGo vs. P/GWGo & 1 & 67.69819 & 310.7287 & $<.001$ \\
Error & 33 & 0.21787 & & \\
\hline
\end{tabular}

\section{Table C2}

Planned contrasts on mean slopes of saccade amplitudes. Road signs are Stop (S), Priority $(P)$ and Give Way (GW).

\begin{tabular}{llrrrr} 
& & Deg & Avg & $F$ & $p$ \\
\cline { 2 - 6 } Before & S vs. P & 1 & 0.00112 & 1.60648 & 0.21386 \\
& Error & 33 & 0.00070 & & \\
\cline { 2 - 6 } & S/P vs. GW & 1 & 0.02954 & 41.43032 & $<.001$ \\
& Error & 33 & 0.00071 & & \\
\hline After & S vs. P & 1 & 0.00916 & 8.73856 & 0.00571 \\
& Error & 33 & 0.00105 & & \\
\cline { 2 - 6 } & GW Go vs. GW NoGo & 1 & 0.00028 & 0.30771 & 0.58283 \\
& Error & 33 & 0.00090 & & \\
\cline { 2 - 6 } & S vs. GWGo/GWNoGo & 1 & 0.02104 & 28.77295 & $<.001$ \\
& Error & 33 & 0.00073 & & \\
\hline
\end{tabular}

Table C3

Planned contrasts on mean values of fixation durations. Road signs are Stop (S), Priority (P) and Give Way (GW).
Lemonnier, S., Brémond, R. \& Baccino, T. (2014) Eye movements in a decision-making driving task

\begin{tabular}{llrrrr} 
& & Deg & Avg & $F$ & $p$ \\
\hline \multirow{2}{*}{ Before } & P vs. GW & 1 & 0.02042 & 2.98115 & 0.09359 \\
& Error & 33 & 0.00685 & & \\
\cline { 2 - 5 } & S vs. P/GW & 1 & 0.09943 & 10.95056 & 0.00227 \\
& Error & 33 & 0.00908 & & \\
\hline After & S vs. GW NoGo & 1 & 0.07276 & 3.28384 & 0.07907 \\
& Error & 33 & 0.02216 & & \\
\cline { 2 - 6 } & P vs. GW Go & 1 & 0.32723 & 8.39987 & 0.00662 \\
& Error & 33 & 0.03896 & & \\
\cline { 2 - 6 } & S/GWNoGo vs. P & 1 & 0.75679 & 19.44325 & $<.001$ \\
& Error & 33 & 0.03892 & & \\
\hline
\end{tabular}

\section{Table C4}

Planned contrasts on mean slopes of fixation durations. Road signs are Stop (S), Priority (P) and Give Way (GW). 
Journal of Eye Movement Research

$7(4): 3,1-14$

\section{References}

Ballard, D. H., Hayhoe, M., \& Peltz, J. (1995). Memory representations in natural tasks. Cognitive Neuroscience, 7, 66-80.

Ballard, D. H., Hayhoe, M. M., Li, F., Whitehead, S. D., Frisby, J., Taylor, J., \& Fisher, R. (1992). Hand-eye coordination during sequential tasks [and discussion]. Philosophical Transactions of the Royal Society of London. Series B: Biological Sciences, 337(1281), 331-339.

Brenner, E., \& Smeets, J. B. (2011). Continuous visual control of interception. Human movement science, 30(3), 475-494.

Bruce, N. D., \& Tsotsos, J. K. (2009). Saliency, attention, and visual search: An information theoretic approach. Journal of vision, 9(3), 5 .

Chang, C.-C., \& Lin, C.-J. (2011). LIBSVM: a library for support vector machines. ACM Trans. on Intelligent Systems and Technology, 3(2), 27.

Chapman, P. R., \& Underwood, G. (1998). Visual search of driving situations: danger and experience. Perception, 27(8), 951-64.

Crundall, D., \& Underwood, G. (2011). Chapter 11 - visual attention while driving: Measures of eye movements used in driving research. In B. E. Porter (Ed.), Handbook of traffic psychology (p. 137-148). San Diego: Academic Press.

Donges, E. (1978). A two-level model of driver steering behavior. Human Factors: The Journal of the Human Factors and Ergonomics Society, 20(6), 691-707.

Eccles, J. S., \& Wigfield, A. (2002). Motivational beliefs, values, and goals. Annual review of psychology, 53(1), 109-132.

Follet, B., Le Meur, O., \& Baccino, T. (2010). Modeling visual attention on scenes. Studia Informatica Universalis, 8(4), 157160.

Foulsham, T., Walker, E., \& Kingstone, A. (2011). The where, what and when of gaze allocation in the lab and the natural environment. Vision Research, 51(17), 1920 - 1931.

Geisler, W. S. (2011). Contributions of ideal observer theory to vision research. Vision research, 51(7), 771-781.

Gigerenzer, G., \& Gaissmaier, W. (2011). Heuristic decision making. Annual review of psychology, 62, 451-482.

Goldberg, J. H., \& Kotval, X. P. (1999). Computer interface evaluation using eye movements: methods and constructs. International Journal of Industrial Ergonomics, 24(6), 631-645.

Goodale, M. A. (2011). Transforming vision into action. Vision research, 51(13), 1567-1587.

Greene, M. R., Lui, T., \& Wolfe, J. M. (2012). Reconsidering yarbus: A failure to predict observer's task from eye movement patterns. Vision Research, 62, 1-8.

Hancock, P., Caird, J., Shekhar, S., \& Vercruyssen, M. (1991). Factors influencing drivers' left turn decisions. In Proceedings of the human factors and ergonomics society annual meeting (Vol. 35, p. 1139-1143). San Francisco, CA.
Lemonnier, S., Brémond, R. \& Baccino, T. (2014) Eye movements in a decision-making driving task

Hayhoe, M., \& Ballard, D. H. (2005). Eye movements in natural behavior. Trends in Cognitive Science, 9(4), 188-194.

Hayhoe, M., Shrivastrava, A., Myruczek, R., \& Pelz, J. (2003). Visual memory and motor planning in a natural task. Journal of Vision, 3, 49-63.

Henderson, J. M., \& Hollingworth, A. (1999). The role of fixation position in detecting scene changes across saccades. Psychological Science, 5, 438-443.

Henderson, J. M., Shinkareva, S. V., Wang, J., Luke, S. G., \& Olejarczyk, J. (2013). Predicting cognitive state from eye movements. PloS one, 8(5), e64937.

Howard, C. J., Gilchrist, I. D., Troscianko, T., Behera, A., \& Hogg, D. C. (2011). Task relevance predicts gaze in videos of real moving scenes. Experimental Brain Research, 214(1), 131-137.

Itti, L., \& Koch, C. (2001). Computational modeling of visual attention. Nature Reviews Neuroscience, 2(3), 194-203.

Johnson, V. E. (2013). Revised standards for statistical evidence. Proceedings of the National Academy of Sciences, 110(48), 19313-19317.

Kahneman, D. (2002). Maps of bounded rationality: A perspective on intuitive judgment and choice. Nobel prize lecture, 8, 351-401.

Kingstone, A., Smilek, D., \& Eastwood, J. D. (2008). Cognitive ethology: a new approach for studying human cognition. British Journal of Psychology, 99(3), 317-340.

Koch, C., \& Ullman, S. (1985). Shifts in selective visual attention: towards the underlying neural circuitry. Human Neurobiology, 4, 219-227.

Land, M. F. (2004). Eye movements in daily life. In L. Chalupa \& J. Werner (Eds.), The visual neurosciences (pp. 1357-1368). Cambridge, MA: MIT.

Land, M. F., \& Horwood, J. (1995). Which parts of the road guide steering? Nature, 377, 339-340.

Land, M. F., \& Lee, D. N. (1994, Juin). Where we look when we steer. Nature, 369, 742-744.

Land, M. F., Mennie, N., \& Rusted, J. (1999). The roles of vision and eye movements in the control of activities of daily living. Perception, 28, 1311-1328.

Land, M. F., \& Tatler, B. (2009). Driving. In Looking and acting: vision and eye movements in natural behaviour (pp. 117-141). NY: Oxford University Press.

Li, W., Pich, V., \& Gilbert, C. (2004). Perceptual learning and top-down influences in primary visual cortex. Nature Neuroscience, 7(6), 651-657.

Lipps, M., \& Pelz, J. B. (2004). Yarbus revisited: taskdependent oculomotor behavior. Journal of Vision, 4(8), 115 
Journal of Eye Movement Research

$7(4): 3,1-14$

Maule, A. J., Hockey, G. R. J., \& Bdzola, L. (2000). Effects of time-pressure on decision-making under uncertainty: changes in affective state and information processing strategy. Acta psychologica, 104(3), 283-301.

Mennie, N., Hayhoe, M., \& Sullivan, B. (2007). Look-ahead fixations: anticipatory eye movements in natural tasks. Experimental Brain Research, 179(3), 427-442.

Näätänen, R., \& Summala, H. (1974). A model for the role of motivational factors in drivers decision making. Accident Analysis \& Prevention, 6, 243-261.

Nenkov, G. Y., \& Gollwitzer, P. M. (2012). Pre- versus postdecisional deliberation and goal commitment: The positive effects of defensiveness. Journal of Experimental Social Psychology, 48(1), 106-121.

Rayner, K. (1998). Eye movements in reading and information processing: 20 years of research. Psychological bulletin, $124(3), 372$

Rothkopf, C. A., Ballard, D. H., \& Hayhoe, M. M. (2007). Task and context determine where you look. Journal of Vision, $7(14), 1-20$

Salvucci, D. D., \& Gray, R. (2004). A two-point visual control model of steering. Perception, 33(10), 1233-1248.

Sprague, N., \& Ballard, D. H. (2003). Eye movements for reward maximization. In Advances in neural information processing systems (vol. 16). MIT-Press.
Lemonnier, S., Brémond, R. \& Baccino, T. (2014) Eye movements in a decision-making driving task

Summerfield, C., \& Egner, T. (2009). Expectation (and attention) in visual cognition. Trends in Cognitive Science, 13(9), 403-409.

Summerfield, C., Tsetsos, K., Van Maanen, L., Dreher, J.-C., \& Ruff, C. (2012). Building bridges between perceptual and economic decision-making: neural and computational mechanisms. Frontiers in neuroscience, 6, 6 .

Svenson, O. (1992). Differentiation and consolidation theory of human decision making: A frame of reference for the study of pre-and post-decision processes. Acta Psychologica, 80(1), 143-168.

Svenson, O., Salo, I., \& Lindholm, T. (2009). Post-decision consolidation and distortion of facts. Judgment and decision making, 4(5), 397-407.

Tatler, B., Hayhoe, M., Land, M. F., \& Ballard, D. H. (2011). Eye guidance in natural vision: Reinterpreting salience. Journal of Vision, 11(5), 1-23.

Vapnik, V. (1998). Statistical learning theory. New York: WileyInterscience.

Wallis, G., Chatziastros, A., Tresilian, J., \& Tomasevic, N. (2007). The role of visual and nonvisual feedback in a vehicle steering task. Journal of Experimental Psychology: Human Perception and Performance, 33(5), 1127.

Yarbus, A. L. (1967). Eye movements and vision. New York: Plenum. 